\title{
An inverse approach to convex ordered median problems in trees*
}

\author{
Elisabeth Gassner ${ }^{\dagger}$
}

\begin{abstract}
The convex ordered median problem is a generalization of the median, the $k$-centrum or the center problem. The task of the associated inverse problem is to change edge lengths at minimum cost such that a given vertex becomes an optimal solution of the location problem, i.e., an ordered median. It is shown that the problem is NP-hard even if the underlying network is a tree and the ordered median problem is convex and either the vertex weights are all equal to 1 or the underlying problem is the $k$-centrum problem. For the special case of the inverse unit weight $k$-centrum problem a polynomial time algorithm is developed.
\end{abstract}

Keywords: Location problem, inverse optimization, ordered median, complexity analysis, $k$-centrum

\section{Introduction}

This paper deals with an inverse location problem. Classical location problems are given by a set of $n$ clients whose location is known and whose importance is modelled by weights. The task is to locate a facility such that the facility is close to the clients. The quality of the location can be measured in different ways. The most famous problems are the 1-median where the quality of a location is measured by the sum of weighted distances to the clients and the 1-center problem where the objective function is the maximum among the weighted distances to the clients. Recently, Nickel and Puerto [11] introduced a unified objective function, the ordered median

${ }^{*}$ This research has been supported by the Austrian Science Fund (FWF) Project P18918-N18.

${ }^{\dagger}$ Technische Universität Graz, Institut für Mathematik B, Steyrergasse 30, 8010 Graz, Austria, gassner@opt.math.tu-graz.ac.at 
function, which contains most of the classical objective functions as special cases.

We apply an inverse approach to ordered median problems. Here the goal is to change values of parameters such that a given location becomes an optimal one. In case of inverse location problems on graphs there are two parameter sets that can be changed: the vertex weights and the edge lengths. Cai et al. [4] considered the problem of changing vertex weights within certain limits such that a given point becomes 1-center. Although the 1-center problem is solvable in polynomial time the authors showed that its inverse in NP-hard. Burkard et al. [1,2] investigated the inverse 1-median problem with variable vertex weights and proved that the problem is solvable by a greedy-type algorithm in $\mathcal{O}(n \log n)$ time if the underlying network is a tree or the location problem is defined in the plane (where distances are measured by the Manhattan or Tchebychev metric) and in $\mathcal{O}\left(n^{2}\right)$ time on cycles. Recently, Burkard et al. [3] solved the inverse Fermat-Weber problem with variable vertex weights in linear time.

Instead of changing vertex weights one may also consider the problem of changing edge lengths. In practice edge length model distances or travel times. These parameters may be influenced by introducing new technologies or improving travel connections. However, much less attention was paid to inverse location problems with variable edge lengths (instead of vertex weights). Gassner [7] considered the inverse 1-maxian problem (i.e., the maximization variant of the 1-median problem) and proved NP-hardness even on series-parallel graphs. However, for trees a linear time algorithm is suggested. For a survey of inverse optimization the reader is referred to [9].

Let us start with a formal problem formulation: Let $G=(V, E, w, \ell)$ be a graph with vertex weights $w_{v} \in \mathbb{R}_{+}$(for $v \in V$ ) and edge lengths $\ell_{e} \in \mathbb{R}_{+}$ (for $e \in E$ ) and let $\lambda \in \mathbb{R}_{+}^{n}$ be a vector. Then the ordered median objective value of a point $x \in G$ is given by

$$
f_{\ell}(x)=\sum_{v \in V} \lambda_{\varphi(v)} w_{v} d_{\ell}(v, x)
$$

where $d_{\ell}(v, x)$ is the shortest distance from $v$ to $x$ with respect to $\ell$ and $\varphi: V \rightarrow\{1, \ldots, n\}$ is an ordering of the vertices according to their weighted distances to $x$, i.e., if $w_{v^{\prime}} d_{\ell}\left(v^{\prime}, x\right)<w_{v^{\prime \prime}} d_{\ell}\left(v^{\prime \prime}, x\right)$ then $\varphi\left(v^{\prime}\right)<\varphi\left(v^{\prime \prime}\right)$. Such an ordering is called a feasible permutation. Consider the 1-center problem: Here the objective function is equal to the maximum among the weighted distances, i.e., the weighted distances are sorted and then the maximum among them is multiplied with 1 while all other weighted distanced are multiplied 
with 0 . The ordered median objective function allows to multiply the ordered weighted distances with arbitrary multipliers. However, it sometimes makes sense that larger weighted distances have more influence to the objective value than smaller ones. This requirement can be modelled by choosing a vector $\lambda$ that is monotonically increasing in its components. If this is the case, i.e., $\lambda_{i} \leq \lambda_{i+1}(i=1, \ldots, n-1)$ then the model is called convex. Observe that the 1 -median $(\lambda=(1, \ldots, 1)), k$-centrum $(\lambda=(0, \ldots, 0,1, \ldots, 1)$ with $k 1$ 's $)$ and 1-center $(\lambda=(0, \ldots, 0,1))$ are special convex ordered medians. Ordered median Problems have been treated in [5,10-12]. Kalcsics et al. [10] investigated the ordered median problem from an algorithmic point of view and showed that the ordered median problem can be solved in $\mathcal{O}\left(m n^{2} \log n\right)$ time on general graphs. If the underlying network is a tree, then this algorithm runs in $\mathcal{O}\left(n^{3} \log n\right)$ time. Moreover, algorithms for the convex ordered median problem $\left(\mathcal{O}\left(n \log ^{2} n\right)\right.$ time $)$ and $k$-centrum problem $(\mathcal{O}(n \log n)$ time $)$ on trees are suggested.

Let $G=(V, E, w, \ell)$ together with $\lambda \in \mathbb{R}_{+}^{n}$ be an instance of the ordered median problem. In addition we are given a vertex $x \in V$, a bound $u_{e}^{+} \in \mathbb{R}_{+}$ for increasing and a bound $u_{e}^{-} \in \mathbb{R}_{+}$for decreasing the length of edge $e \in E$. Then the task of the inverse ordered median problem (InvP for short) is to find edge length modifications $(p, q)$ such that

- $(p, q) \in \Delta$ with $\Delta=\left\{(p, q) \in \mathbb{R}^{2 n} \mid 0 \leq p_{e} \leq u_{e}^{+}, 0 \leq q_{e} \leq u_{e}^{-} \forall e \in E\right\}$,

- $x$ is ordered median with respect to edge lengths $\tilde{\ell}_{e}=\ell_{e}+p_{e}-q_{e}$ (for $e \in E)$, and

- the difference between $\ell$ and $\tilde{\ell}$ measured by the $\ell_{1}$-norm is minimized.

Obviously, every optimal solution $(p, q)$ satisfies the orthogonality condition $p_{e} q_{e}=0$ for all $e \in E$. Therefore, $\|\ell-\tilde{\ell}\|_{1}=\sum_{v \in V}\left(p_{e}+q_{e}\right)$ holds for an optimal solution.

Organization of this paper. It turns out that InvP is in general NPhard even for several restricted cases. Both NP-hardness result concern InvP where the underlying ordered median problem is convex and the network is a tree. We show that $\operatorname{InvP}$ is hard even if the vertex weights are all equal to 1 or if the convex ordered median problem is the $k$-centrum problem.

These negative results motivated us to investigate the inverse unit-weight $k$-centrum problem which is still a generalization of the 1-median and 1center problem. In Section 2 we give a short survey about convex ordered median problems on trees. Section 3 provides the already mentioned NPhardness results and in Section 4 we suggest a polynomial time algorithm for the inverse unit-weight $k$-centrum problem on trees. 
Notation. Throughout this paper we will use the following notation: $T=(V, E)$ is a tree. We write $x \in T$ if $x$ coincides with a vertex or lies on an edge of $T$. Let $x, v \in V$ then $P(x, v)$ denotes the unique path from $x$ to $v$. Let $x \in V$ then the set of subtrees that result from deleting vertex $x$ is denoted by $\mathcal{T}(x)$. If $x \in T$ lies in the interior of edge $e \in E$ then $\mathcal{T}(x)$ is the set of subtrees that result from deleting the edge $e$.

Let $T^{\prime}$ be a subtree of $T$ then $T-T^{\prime}$ denotes the graph that is induced by all vertices not in $T^{\prime}$. If $T^{\prime} \in \mathcal{T}(x)$ then $T^{\prime}$ and $T-T^{\prime}$ can be considered as directed trees with root $x$. For a vertex $v$ in a rooted tree, $\Gamma(v)$ denotes the set of children, $\operatorname{outdeg}(v)=|\Gamma(v)|$ is the outdegree and $T_{v}$ is the subgraph spanned by all successors including vertex $v$.

\section{Convex ordered median problems on trees}

From now on we assume that $G=T$ is a tree and $\lambda$ is monotonically increasing in its components. Kalcsics et al. [10] showed that $f_{\ell}(x)$ is then convex along a path. Hence, a point $x \in G$ is ordered median if and only if the derivatives of $f_{\ell}(x)$ in the direction of its neighbours are nonnegative. We will use the following formulation of the optimality criterion:

Theorem 2.1 (Optimality criterion, Reformulation of [10]). Given an instance of the convex ordered median problem with tree $T=(V, E, w, \ell)$ and vector $\lambda \in \mathbb{R}^{n}$. Then $x \in T$ is ordered median if and only if for each $T^{\prime} \in \mathcal{T}(x)$ there exists a feasible permutation $\varphi^{\prime}$ such that

$$
\sum_{v \in T^{\prime}} \lambda_{\varphi^{\prime}(v)} w_{v} \leq \frac{1}{2} \sum_{v \in V} \lambda_{\varphi^{\prime}(v)} w_{v} .
$$

For the special case of the 1-median problem, the above optimality criterion (1) is independent of the edge lengths because $\lambda_{i}=1$ holds for $i=1, \ldots, n$ (cf. Goldman [8]). In case of the unweighted 1-center problem, it is easy to see that the above optimality criterion is equivalent to the so-called midpoint property, i.e., the 1-center lies on the midpoint of a longest path. The following lemma is straightforward:

Lemma 2.2. Let $x \in T$, then there exists at most one subtree $T^{\prime} \in \mathcal{T}(x)$ that does satisfy the optimality criterion (1).

Let us turn our attention to the unit-weight $k$-centrum problem. Observe that the $k$-centrum problem is a generalization of the 1-median as well as of the 1-center problem. 
Definition 2.3. Let $T=(V, E, \ell)$ be a tree with edge lengths $\ell$. Furthermore, let $x \in T, L \in \mathbb{R}$ and $\tilde{T}$ a subtree of $T$. Then we define the number of vertices in $\tilde{T}$ whose weighted distance to $x$ is more than $L$ and the number of vertices in $\tilde{T}$ whose weighted distance to $x$ is equal to $L$, i.e.,

$$
\begin{aligned}
& \alpha_{\ell}(\tilde{T}, L, x)=\left|\left\{v \in \tilde{T} \mid d_{\ell}(x, v)>L\right\}\right|, \\
& \beta_{\ell}(\tilde{T}, L, x)=\left|\left\{v \in \tilde{T} \mid d_{\ell}(x, v)=L\right\}\right| .
\end{aligned}
$$

A real number $L$ is called critical for $x \in T$ and edge length vector $\ell$ if

$$
\alpha_{\ell}(T, L, x)<k \quad \text { and } \quad \alpha_{\ell}(T, L, x)+\beta_{\ell}(T, L, x) \geq k .
$$

Observe that the critical length $L$ is equal to the distance from $x$ to the $k$-farthest vertex.

In a next step we investigate the optimality criterion for the unit weight $k$-centrum problem:

$$
\sum_{v \in V} \lambda_{\varphi(v)}=k
$$

holds for every feasible permutation $\varphi$. Now let $x \in V$ and let $L$ be the critical length for $x$ and edge length $\ell$. If $d_{\ell}(x, v)>L\left(d_{\ell}(x, v)<L\right)$ then $\lambda_{\varphi(v)}=1\left(\lambda_{\varphi(v)}=0\right)$ and exactly $k-\alpha_{\ell}(T, L, x)$ vertices with $d_{\ell}(x, v)=L$ satisfy $\lambda_{\varphi(v)}=1$ for every feasible $\varphi$. Consider a subtree $T^{\prime} \in \mathcal{T}(x)$. We are interested in the smallest number $b$ of vertices $v \in T^{\prime}$ with $d_{\ell}(v, x)=L$ and $\lambda_{\varphi(v)}=1$ such that $\varphi$ is a feasible permutation. If $\alpha_{\ell}(T, L, x)+\beta_{\ell}(T-$ $\left.T^{\prime}, L, x\right) \geq k$ then $b=0$ and otherwise $b=k-\alpha_{\ell}(T, L, x)-\beta_{\ell}\left(T-T^{\prime}, L, x\right)$ holds. Hence, $b=\max \left\{0, k-\alpha_{\ell}(T, L, x)-\beta_{\ell}\left(T-T^{\prime}, L, x\right)\right\}$ and consequently

$$
\begin{aligned}
\min _{\substack{\varphi \text { is } \\
\text { feasible }}} \sum_{v \in T^{\prime}} \lambda_{\varphi(v)} & =\alpha_{\ell}\left(T^{\prime}, L, x\right)+b \\
& =\max \left\{\alpha_{\ell}\left(T^{\prime}, L, x\right), k-\left(\alpha_{\ell}\left(T-T^{\prime}, L, x\right)+\beta_{\ell}\left(T-T^{\prime}, L, x\right)\right)\right\}
\end{aligned}
$$

holds. These observations imply the following optimality criterion for the $k$-centrum problem:

Theorem 2.4. Let $T=(V, E, \ell)$ be a tree with edge lengths $\ell$ and unit weights. Then $x \in T$ is $k$-centrum if and only if

$$
\begin{aligned}
\alpha_{\ell}\left(T^{\prime}, L, x\right) & \leq \frac{k}{2}, \text { and } \\
\alpha_{\ell}\left(T-T^{\prime}, L, x\right)+\beta_{\ell}\left(T-T^{\prime}, L, x\right) & \geq \frac{k}{2}
\end{aligned}
$$

hold for the critical length $L$. 
The above optimality criterion has the following meaning: Let $x \in T$ and let $L$ be the critical length ( $k$-farthest distance to $x$ ). Then $x$ is $k$-centrum if and only if in every subtree $T^{\prime} \in \mathcal{T}(x)$ there are at most $\frac{k}{2}$ vertices whose distance to $x$ is more than $L$ while there are at least $\frac{k}{2}$ vertices not in $T^{\prime}$ whose distance to $x$ is at least $L$. Consider the 1-center problem, which is equal to the 1-centrum problem. Then the above optimality criterion is equivalent to the following conditions: $\alpha_{\ell}\left(T^{\prime}, L, x\right) \leq \frac{1}{2}$ and hence $\alpha_{\ell}\left(T^{\prime}, L, x\right)=0$ means that there are at least two vertices with maximum distance to $x$. The second condition means that there are two vertices with maximum distance to $x$ that lie in different subtrees of $x$. But then these two vertices are endpoints of a longest path. Therefore, we get that $x$ is 1-center if and only if $x$ lies on the midpoint of a longest path.

\section{NP-hardness results}

In this section, we show that InvP is NP-hard even if all vertex weights are equal to 1 (Subsection 3.1) or if the underlying ordered median problem is the $k$-centrum problem (Subsection 3.2).

\subsection{The special case of unit weights}

Theorem 3.1. The inverse ordered median problem is NP-hard even for the convex case on trees and for unit weights.

Proof. Consider the pairwise different Partition Problem which is defined as follows: Given a set of $n$ pairwise different integers $\left\{a_{i} \mid i=1, \ldots, n\right\}$ and let $2 B=\sum_{i=1}^{n} a_{i}$. The Partition Problem asks whether there exists a subset $X \subset\{1, \ldots, n\}$ of indices with $\sum_{i \in X} a_{i}=B=\sum_{i \notin X} a_{i}$. The Partition Problem is known to be NP-hard [6] and it can easily be shown that the problem remains NP-hard even if we require that the integers are pairwise different. Moreover, we may assume without loss of generality that $a_{i} \leq B$ holds for $i=1, \ldots, n$ because otherwise the Partition Problem is trivial.

Given an instance I(Partition) of the pairwise different Partition Problem with $1 \leq a_{1}<a_{2}<\cdots<a_{n}$, we construct an instance of InvP as follows: The tree $T=(V, E)$ consists of $2 n+2$ vertices and $2 n+1$ edges:

$$
\begin{aligned}
& V=\left\{x, y_{0}\right\} \cup\left\{x_{i}, y_{i} \mid i=1, \ldots, n\right\}, \\
& E=\left\{\left(x, y_{0}\right)\right\} \cup\left\{\left(x, x_{i}\right),\left(y_{0}, y_{i}\right) \mid i=1, \ldots, n\right\} .
\end{aligned}
$$


The edge lengths and bounds for decreasing are given by

$$
\ell_{e}=\left\{\begin{array}{ll}
1 & \text { if } e=\left(x, y_{0}\right) \\
(B-1) a_{i} & \text { if } e=\left(x, x_{i}\right) \\
B a_{i}-1 & \text { if } e=\left(y_{0}, y_{i}\right)
\end{array} \quad \text { and } \quad u_{e}^{-}= \begin{cases}a_{i} & \text { if } e=\left(y_{0}, y_{i}\right) \\
0 & \text { otherwise }\end{cases}\right.
$$

Finally, $u_{e}^{+}=0$ for all $e \in E, \lambda$ is given by

$$
\lambda=\left(0,0, \alpha_{1}, \beta_{1}, \alpha_{2}, \beta_{2}, \ldots, \alpha_{n}, \beta_{n}\right)
$$

with $\alpha_{i}=(B-1) a_{i}$ and $\beta_{i}=B a_{i}$ for $i=1, \ldots, n$. Vertex $x$ should become an ordered median.

Let us start with some observations concerning the constructed instance $\mathrm{I}(\operatorname{InvP})$ : Since $a_{i} \leq a_{i+1}-1$ the vector $\lambda$ is monotonically increasing in its components and therefore the instance is convex.

Consider any length vector $\tilde{\ell}=\ell+p-q$ with $(p, q) \in \Delta$. Then $d_{\ell}\left(x, x_{i}\right)=$ $d_{\tilde{\ell}}\left(x, x_{i}\right)=(B-1) a_{i}$ for $i=1, \ldots, n$ and $d_{\ell}\left(x, y_{0}\right)=d_{\tilde{\ell}}\left(x, y_{0}\right)=1$. Moreover,

$d_{\tilde{\ell}}\left(x, x_{i}\right)=d_{\ell}\left(x, y_{i}\right)-u_{\left(y_{0}, y_{i}\right)}^{-} \leq d_{\tilde{\ell}}\left(x, y_{i}\right) \leq B a_{i}<(B-1) a_{i+1}=d_{\tilde{\ell}}\left(x, x_{i+1}\right)$

holds for all $i=1, \ldots, n$. Therefore, $d_{\tilde{\ell}}\left(x, y_{0}\right)<d_{\tilde{\ell}}\left(x, x_{1}\right)$ and $d_{\tilde{\ell}}\left(x, x_{i}\right) \leq$ $d_{\tilde{\ell}}\left(x, y_{i}\right)<d_{\tilde{\ell}}\left(x, x_{i+1}\right) \leq d_{\tilde{\ell}} d\left(x, y_{i+1}\right)$ hold for $i=1, \ldots, n-1$. Let $\varphi$ be any feasible permutation for $\tilde{\ell}$ then $\varphi(x)=1, \varphi\left(y_{0}\right)=2, \varphi\left(x_{i}\right) \in\{2 i+1,2 i+2\}$ and $\varphi\left(y_{i}\right) \in\{2 i+1,2 i+2\}$. Especially $\varphi^{*}$ with $\varphi^{*}(x)=1, \varphi^{*}\left(y_{0}\right)=2$, $\varphi^{*}\left(x_{i}\right)=2 i+1$ and $\varphi^{*}\left(y_{i}\right)=2 i+2$ is feasible for $\tilde{\ell}$. The permutation $\varphi^{*}$ makes sure that every subtree $\tilde{T} \in \mathcal{T}(x)$ with $y_{0} \notin \tilde{T}$ satisfies the optimality criterion. Let $T^{\prime} \in \mathcal{T}(x)$ with $y_{0} \in T^{\prime}$. Then the above observation implies that $x$ is ordered median with respect to $\tilde{\ell}$ if and only if the optimality criterion for $T^{\prime} \in \mathcal{T}(x)$ is satisfied. The optimality criterion is satisfied for $T^{\prime}$ if and only if the criterion is satisfied for the following feasible permutation $\varphi^{\prime}$ (because this permutation minimizes the left-hand side of $(1)$ ): $\varphi^{\prime}(x)=1$, $\varphi^{\prime}\left(y_{0}\right)=2$ and

$$
\varphi^{\prime}\left(x_{i}\right)= \begin{cases}2 i+1 & d_{\tilde{\ell}}\left(x, x_{i}\right)<d_{\tilde{\ell}}\left(x, y_{i}\right) \\ 2 i+2 & d\left(x, x_{i}\right)=d\left(x, y_{i}\right)\end{cases}
$$

and

$$
\varphi^{\prime}\left(y_{i}\right)= \begin{cases}2 i+1 & d_{\tilde{\ell}}\left(x, x_{i}\right)=d_{\tilde{\ell}}\left(x, y_{i}\right) \\ 2 i+2 & d\left(x, x_{i}\right)<d\left(x, y_{i}\right)\end{cases}
$$


We will show that there exists a partition of I(Partition) if and only if $\mathrm{I}(\operatorname{InvP})$ has a feasible solution with objective value at most $B$.

$\Longrightarrow$ : Assume that there exists a partition $X$ with $\sum_{i \in X} a_{i}=B$. Set

$$
q_{e}= \begin{cases}u_{e}^{-}=a_{i} & \text { if } e=\left(y_{0}, y_{i}\right) \text { and } i \in X \\ 0 & \text { otherwise }\end{cases}
$$

Then we have $d_{\tilde{\ell}}\left(x, x_{i}\right)=d_{\tilde{\ell}}\left(x, y_{i}\right)$ if and only if $i \in X$ and

$$
\sum_{v \in T^{\prime}} \lambda_{\varphi^{\prime}(v)}=\sum_{i \in X} \alpha_{i}+\sum_{i \notin X} \beta_{i}=2 B^{2}-B=\sum_{i \notin X} \alpha_{i}+\sum_{i \in X} \beta_{i}=\sum_{v \notin T^{\prime}} \lambda_{\varphi^{\prime}(v)} .
$$

Hence, $x$ is ordered median with respect to $\tilde{\ell}$. The objective value of the modification is equal to $\sum_{e \in E} q_{e}=\sum_{i \in X} a_{i}=B$.

$\Longleftarrow$ : Assume that $(p, q)$ is a feasible solution of $\mathrm{I}(\operatorname{InvP})$ with objective value at most $B$ such that there exists an edge $e^{\prime}=\left(y_{0}, y_{i}\right)$ with $0<q_{e^{\prime}}<$ $u_{e^{\prime-}}=a_{i}$. Then $\varphi^{\prime}$ fulfills $\varphi^{\prime}\left(x_{i}\right)=2 i+1$ and $\varphi^{\prime}\left(y_{i}\right)=2 i+2$. Since $x$ is ordered median, the permutation $\varphi^{\prime}$ satisfies the optimality condition for $T^{\prime}$. However, if we set $\tilde{q}_{e^{\prime}}=0$ and $\tilde{q}_{e}=q_{e}$ for all $e \neq e^{\prime}$ then $\varphi^{\prime}$ is still a feasible permutation for the new length vector and hence $x$ is also ordered median with respect to the new length modification but the objective value of $(p, \tilde{q})$ is smaller than the objective value of $(p, q)$. Therefore, we conclude that there exists a feasible solution $(p, q)$ of $\mathrm{I}(\operatorname{InvP})$ with cost at most $B$ and $q_{e} \in\left\{0, u_{e}^{-}\right\}$for every $e \in E$.

Consider the set $X=\left\{i \mid q_{\left(y_{0}, y_{i}\right)}=u_{\left(y_{0}, y_{i}\right)}^{-}\right\}$. Then $d_{\tilde{\ell}}\left(x, x_{i}\right)=d_{\tilde{\ell}}\left(x, y_{i}\right)$ holds if and only if $q_{\left(y_{0}, y_{i}\right)}=u_{\left(y_{0}, y_{i}\right)}^{-}$and hence $i \in X$. Simple calculation yield $\sum_{i \in X} a_{i} \geq B$ because $x$ is 1-median. On the other hand, the objective value of the modification $\sum_{e \in E} q_{e}=\sum_{i \in X} a_{i} \leq B$. Hence, $\sum_{i \in X} a_{i}=B$ and $X$ is a partition.

\subsection{The special case of the $k$-centrum problem}

Theorem 3.2. The inverse ordered median problem is NP-hard even if the underlying location problem is the $k$-centrum problem.

Proof. Consider the cardinality constrained Partition Problem where a set of $n$ integers $\left\{a_{i} \mid i=1, \ldots, n\right\}$ is given with $2 B=\sum_{i=1}^{n} a_{i}$. Moreover, an integer $1 \leq k^{\prime} \leq n$ is given. The cardinality constrained Partition Problem asks whether there exists a subset $X \subset\{1, \ldots, n\}$ of indices of cardinality $k^{\prime}$ with $\sum_{i \in X} a_{i}=B$. The cardinality constrained Partition Problem is NP-hard unless $\mathrm{P}=$ NP because otherwise one could solve $\mathcal{O}(n)$ cardinality 
constrained Partition Problems in order to get a solution of the Partition Problem.

Given an instance I(CardPart) of the cardinality constrained Partition Problem, we construct an instance of InvP as follows: The tree $T=(V, E)$ consists of $n+3$ vertices $V=\left\{x, x_{1}\right\} \cup\left\{y_{i} \mid i=0, \ldots, n\right\}$ and edge set $E=\left\{\left(x, x_{1}\right),\left(x, y_{0}\right)\right\} \cup\left\{\left(y_{0}, y_{i}\right) \mid i=1, \ldots, n\right\}$.

The vertex weights are equal to $w_{y_{i}}=a_{i}$ for $i=1, \ldots, n, w_{x_{1}}=B$ and $w_{v}=0$ otherwise. The edge lengths and bounds for decreasing are given by

$$
\ell_{e}= \begin{cases}a_{i}+\frac{B}{a_{i}}-1 & \text { if } e=\left(y_{0}, y_{i}\right), i=1, \ldots, n \\ 1 & \text { otherwise }\end{cases}
$$

and

$$
u_{e}^{-}= \begin{cases}a_{i} & \text { if } e=\left(y_{0}, y_{i}\right) \\ 0 & \text { otherwise }\end{cases}
$$

Finally, $u_{e}^{+}=0$ for all $e \in E$ and $x$ should become $k$-centrum for $k=$ $n+1-k^{\prime}$. It will be shown that there exists a solution of I(CardPart) if and only if there exists a feasible solution for the constructed instance I(InvP) with objective value at most $B$.

Observe that $w_{y_{i}} d_{\tilde{\ell}}\left(y_{i}, x\right) \geq B=w_{x_{1}} d_{\tilde{\ell}}\left(x_{1}, x\right)$ holds for all $i=1, \ldots, n$ and $w_{y_{i}} d_{\tilde{\ell}}\left(y_{i}, x\right)=w_{x_{1}} d_{\tilde{\ell}}\left(x_{1}, x\right)$ if and only if $q_{\left(y_{0}, y_{i}\right)}=a_{i}$. Hence, for every $(p, q) \in \Delta$ and $\tilde{\ell}=\ell+p-q$ there exists a feasible permutation $\varphi$ such that $\lambda_{\varphi\left(x_{1}\right)}=0$ and hence the optimality condition for $T^{\prime} \in \mathcal{T}(x)$ with $x_{1} \in T^{\prime}$ is satisfied. Therefore, $x$ is $k$-centrum if and only if the optimality condition for $T^{\prime \prime} \in \mathcal{T}(x)$ with $y_{0} \in T^{\prime \prime}$ is satisfied. However, this optimality condition is satisfied if and only if there are $n-(k-1)$ vertices in $T^{\prime \prime}$ with $\lambda_{\varphi\left(y_{i}\right)}=0$ whose weights sum up to at least $B$. Hence, $x$ is $k$-centrum if and only if there exists a subset $Y \subset\{1, \ldots, n\}$ of $k^{\prime}=n-(k-1)$ indices such that $q_{\left(y_{0}, y_{i}\right)}=u_{\left(y_{0}, y_{i}\right)}^{-}=a_{i}$ and $\sum_{i \in Y} w_{y_{i}}=\sum_{i \in Y} a_{i} \geq B$. If $(p, q)$ is feasible then there also exists a feasible solution where only those edges in $T^{\prime \prime}$ are modified that are incident to a vertex in $Y$. Therefore, if there exists a feasible solution of $\mathrm{I}(\operatorname{InvP})$ with $\sum_{e \in E} q_{e} \leq B$ then there exists a subset $Y \subset\{1, \ldots, n\}$ of $k^{\prime}=n-(k-1)$ indices such that $\sum_{i \in Y} w_{y_{i}}=\sum_{i \in Y} a_{i} \geq B$ and $\sum_{e \in E} p_{e}=\sum_{i \in Y} a_{i} \leq B$, i.e., there exists a partition $Y$ or cardinality $k^{\prime}$. On the other hand, if there exists a partition $X$ then set $q_{e}=u_{e}^{-}$for all $e=\left(y_{0}, y_{i}\right)$ and $i \in X$ which yields a feasible solution with total cost of at most $B$. 


\section{Inverse unit-weight $k$-centrum problem on trees}

In the previous section, we have seen that InvP is NP-hard even if all weights are equal to 1 or the underlying location problem is the $k$-centrum problem. In this section a polynomial time algorithm for the inverse unit-weight $k$ centrum (InvKC for short) is developed.

Recall that the task of InvP is to minimally change the edge lengths such that a given vertex $x \in V$ becomes 1-median. If the underlying ordered median problem is the unit-weight $k$-centrum problem, then $x \in V$ is 1 median with respect to edge lengths $\tilde{\ell}$ if and only if (2) and (3) hold for the critical length $L$. In order to make notation simple, we will write $\alpha_{\tilde{\ell}}(\tilde{T}, L)$ $\left(\beta_{\tilde{\ell}}(\tilde{T}, L)\right.$, resp. $)$ instead of $\alpha_{\tilde{\ell}}(\tilde{T}, L, x)\left(\beta_{\tilde{\ell}}(\tilde{T}, L, x)\right.$, resp. $)$ because we always refer to the vertex $x$ which should become $k$-centrum. Then InvKC is to solve

$$
\begin{array}{lll}
\min _{p, q, L} & \sum_{e \in E}\left(p_{e}+q_{e}\right) & \\
\text { s.t. } & \alpha_{\tilde{\ell}}(\tilde{T}, L) \leq \frac{k}{2} & \\
& \alpha_{\tilde{\ell}}(T-\tilde{T}, L)+\beta_{\tilde{\ell}}(T-\tilde{T}, L) \geq \frac{k}{2} & \text { for all } \tilde{T} \in \mathcal{T}(x) \\
& \alpha_{\tilde{\ell}}(T, L)<k \text { and } \alpha_{\tilde{\ell}}(T, L)+\beta_{\tilde{\ell}}(T, L) \geq k & \\
& \tilde{\ell}=\ell+p-q,(p, q) \in \Delta &
\end{array}
$$

Recall that due to Lemma 2.2 there exists at most one subtree $T^{\prime} \in \mathcal{T}(x)$ that does not satisfy (2) and (3) for length vector $\ell$. Assume that $T^{\prime} \in \mathcal{T}(x)$ is this unique subtree, then we define the problem $\operatorname{Relax}\left(T^{\prime}\right)$ as follows:

$$
\begin{array}{ll}
\min _{p, q, L} & \sum_{e \in E}\left(p_{e}+q_{e}\right) \\
\text { s.t. } & \alpha_{\tilde{\ell}}\left(T^{\prime}, L\right) \leq \frac{k}{2} \text { and } \alpha_{\tilde{\ell}}\left(T-T^{\prime}, L\right)+\beta_{\tilde{\ell}}\left(T-T^{\prime}, L\right) \geq \frac{k}{2} \\
& \tilde{\ell}=\ell+p-q,(p, q) \in \Delta
\end{array}
$$

$\operatorname{Relax}\left(T^{\prime}\right)$ is indeed a relaxation of $\operatorname{InvKC}$. Moreover, $\operatorname{Relax}\left(T^{\prime}\right)$ has an optimal solution $\left(p^{*}, q^{*}, L^{*}\right)$ such that $p_{e}^{*}=0$ for all $e \in T^{\prime}$ and $q_{e}^{*}=0$ for all $e \in T-T^{\prime}$.

Theorem 4.1. Assume that $T^{\prime} \in \mathcal{T}(x)$ contradicts the optimality criterion for length vector $\ell$ and let $\left(p^{*}, q^{*}, L^{*}\right)$ be an optimal solution of Relax $\left(T^{\prime}\right)$. Then $\left(p^{*}, q^{*}\right)$ is an optimal solution of InvKC. 
Proof. Since $\operatorname{Relax}\left(T^{\prime}\right)$ is a relaxation of $\operatorname{InvKC}$, we have to show that $\left(p^{*}, q^{*}\right)$ is feasible for InvKC, i.e., $x$ is $k$-centrum with respect to $\tilde{\ell}=$ $\ell+p^{*}-q^{*}$.

The proof splits into three parts: In the first part it is shown that there exists at least one edge whose length is changed in $\left(p^{*}, q^{*}, L^{*}\right)$. In the second part we define a real number $L^{\prime}$ and prove two inequalities and in the third part it is shown that $L^{\prime}$ is the critical length for $\tilde{\ell}=\ell+p^{*}-q^{*}$ and the optimality criteria (2) and (3) are satisfied with respect to $\tilde{\ell}$ and $L^{\prime}$ for every subtree of $\mathcal{T}(x)$. This implies that $x$ is $k$-centrum with respect to $\tilde{\ell}$.

1. First we prove that $p_{e}^{*}=q_{e}^{*}=0$ (for all $e \in E$ ) cannot be an optimal solution of $\operatorname{Relax}\left(T^{\prime}\right)$ : Assume the contrary, then $\tilde{\ell}=\ell$ holds. Let $L^{\prime}$ be the critical length with respect to $\ell=\tilde{\ell}$. Since $T^{\prime}$ contradicts the optimality criterion either (2) or (3) is not satisfied for $\ell$ and the critical length $L^{\prime}$. If $\alpha_{\ell}\left(T^{\prime}, L^{\prime}\right)>\frac{k}{2}$ then $L^{\prime}<L^{*}$ because $\alpha_{\ell}\left(T^{\prime}, L^{*}\right) \leq \frac{k}{2}$ which implies $\alpha_{\ell}\left(T-T^{\prime}, L^{*}\right)+\beta_{\ell}\left(T-T^{\prime}, L^{*}\right) \leq \alpha_{\ell}\left(T-T^{\prime}, L^{\prime}\right)$. Moreover, the fact that $L^{\prime}$ is the critical length implies that $\alpha_{\ell}\left(T-T^{\prime}, L^{\prime}\right)<\frac{k}{2}$ because $\alpha_{\ell}\left(T^{\prime}, L^{\prime}\right)+\alpha_{\ell}\left(T-T^{\prime}, L^{\prime}\right)=\alpha_{\ell}\left(T, L^{\prime}\right)<k$. Hence, we get

$$
\alpha_{\ell}\left(T-T^{\prime}, L^{*}\right)+\beta_{\ell}\left(T-T^{\prime}, L^{*}\right) \leq \alpha_{\ell}\left(T-T^{\prime}, L^{\prime}\right)<\frac{k}{2}
$$

which contradicts the feasibility of $\left(p^{*}, q^{*}, L^{*}\right)$ for $\operatorname{Relax}\left(T^{\prime}\right)$.

For the second case where $\alpha_{\ell}\left(T-T^{\prime}, L^{\prime}\right)+\beta_{\ell}\left(T-T^{\prime}, L^{\prime}\right)<\frac{k}{2}$ holds an analogous chain of arguments holds: $L^{\prime}>L^{*}, \alpha_{\ell}\left(T^{\prime}, L^{\prime}\right)+\beta_{\ell}\left(T^{\prime}, L^{\prime}\right) \leq$ $\alpha_{\ell}\left(T^{\prime}, L^{*}\right)$ and $\alpha_{\ell}\left(T^{\prime}, L^{*}\right)<\frac{k}{2}$. Hence, we get $\alpha_{\ell}\left(T^{\prime}, L^{\prime}\right)+\beta_{\ell}\left(T^{\prime}, L^{\prime}\right) \leq$ $\alpha_{\ell}\left(T^{\prime}, L^{*}\right)<\frac{k}{2}$ which is a contradiction. Therefore, there exists at least one edge whose length is modified in an optimal solution of $\operatorname{Relax}\left(T^{\prime}\right)$.

2. Consider an optimal solution $\left(p^{*}, q^{*}, L^{*}\right)$ of $\operatorname{Relax}\left(T^{\prime}\right)$ where the length of at least one edge is changed. Then $L^{\prime}$ is defined in the following way: If there are modifications in $T^{\prime}$ and $T-T^{\prime}$ then $L^{\prime}=L^{*}$, if there is no modification in $T-T^{\prime}$ then $L^{\prime}=\max \left\{L \mid \alpha_{\tilde{\ell}}\left(T-T^{\prime}, L\right)+\beta_{\tilde{\ell}}\left(T-T^{\prime}, L\right) \geq\right.$ $\left.\frac{k}{2}\right\}$ and if there is no modification in $T^{\prime}$ then $L^{\prime}=\min \left\{L \mid \alpha_{\tilde{\ell}}\left(T^{\prime}, L\right) \leq\right.$ $\left.\frac{k}{2}\right\}$. It will turn out that $L^{\prime}$ is the critical length for $\tilde{\ell}$.

Assume that there is no modification in $T-T^{\prime}$ and consider $\left(p^{*}, q^{*}, L^{\prime}\right)$. Then the maximality of $L^{\prime}$ implies $L^{\prime} \geq L^{*}$ and hence $\alpha_{\tilde{\ell}}\left(T^{\prime}, L^{\prime}\right) \leq$ $\alpha_{\tilde{\ell}}\left(T^{\prime}, L^{*}\right) \leq \frac{k}{2}$ holds. On the other hand $\alpha_{\tilde{\ell}}\left(T-T^{\prime}, L^{\prime}\right)+\beta_{\tilde{\ell}}(T-$ $\left.T^{\prime}, L^{\prime}\right) \geq \frac{k}{2}$ holds by construction. Hence, $\left(p^{*}, q^{*}, L^{\prime}\right)$ is again an opti- 
mal solution of Relax $\left(T^{\prime}\right)$. Moreover, the maximality of $L^{\prime}$ implies

$$
\alpha_{\tilde{\ell}}\left(T-T^{\prime}, L^{\prime}\right)<\frac{k}{2}
$$

because otherwise one could increase $L^{\prime}$.

If there is no modification in $T^{\prime}$ then the minimality of $L^{\prime}$ implies $\alpha_{\tilde{\ell}}\left(T^{\prime}, L^{\prime}\right)+\beta_{\tilde{\ell}}\left(T^{\prime}, L^{\prime}\right)>\frac{k}{2}$ and $\left(p^{*}, q^{*}, L^{\prime}\right)$ is again feasible. Hence, in all three cases $\left(p^{*}, q^{*}, L^{\prime}\right)$ is an optimal solution of $\operatorname{Relax}\left(T^{\prime}\right)$.

In this second part, we finally prove two inequalities:

- $\alpha_{\tilde{\ell}}\left(T-T^{\prime}, L^{\prime}\right)<\frac{k}{2}$ :

Assume that $\alpha_{\tilde{\ell}}\left(T-T^{\prime}, L^{\prime}\right) \geq \frac{k}{2}$. Inequality (4) implies that there exists at least one edge edge $e_{2} \in T-T^{\prime}$ with $p_{e_{2}}^{*}>0$. Consider the new solution $\left(\hat{p}, q^{*}\right)$ which is obtained from $\left(p^{*}, q^{*}\right)$ where $p_{e_{2}}^{*}$ is decreased by some small amount $\epsilon>0$. Let $\hat{\ell}$ be the new length vector. Obviously, the constraint for subtree $T^{\prime}$ is still satisfied because we did not change the modifications of edges in $T^{\prime}$. In order to guarantee that the constraint for $T-T^{\prime}$ is also satisfied, $\epsilon>0$ is chosen in such a way that if $d_{\tilde{\ell}}(x, v)>L^{\prime}$ then $d_{\hat{\ell}}(x, v)>L^{\prime}$ for all $v \in T-T^{\prime}$.

$$
\frac{k}{2} \leq \alpha_{\tilde{\ell}}\left(T-T^{\prime}, L^{\prime}\right) \leq \alpha_{\hat{\ell}}\left(T-T^{\prime}, L^{\prime}\right) \leq \alpha_{\hat{\ell}}\left(T-T^{\prime}, L^{\prime}\right)+\beta_{\hat{\ell}}\left(T-T^{\prime}, L^{\prime}\right) .
$$

Hence, $\left(\hat{p}, q^{*}, L^{\prime}\right)$ is a feasible solution of $\operatorname{Relax}\left(T^{\prime}\right)$ with less cost than $\left(p^{*}, q^{*}, L^{\prime}\right)$ which contradicts its optimality.

- $\alpha_{\tilde{\ell}}\left(T^{\prime}, L^{\prime}\right)+\beta_{\tilde{\ell}}\left(T^{\prime}, L^{\prime}\right) \geq \frac{k}{2}$ : This case is proved in an analogous way as the previous one.

3. $\quad-L^{\prime}$ is the critical length of $x$ for $\tilde{\ell}$, because $\alpha_{\tilde{\ell}}\left(T, L^{\prime}\right)=\alpha_{\tilde{\ell}}\left(T^{\prime}, L^{\prime}\right)+$ $\alpha_{\tilde{\ell}}\left(T-T^{\prime}, L^{\prime}\right)<k$ and $\alpha_{\tilde{\ell}}\left(T, L^{\prime}\right)+\beta_{\tilde{\ell}}\left(T, L^{\prime}\right)=\alpha_{\tilde{\ell}}\left(T^{\prime}, L^{\prime}\right)+\beta_{\tilde{\ell}}\left(T^{\prime}, L^{\prime}\right)+$ $\alpha_{\tilde{\ell}}\left(T-T^{\prime}, L^{\prime}\right)+\beta_{\tilde{\ell}}\left(T-T^{\prime}, L^{\prime}\right) \geq k$.

- The optimality condition for every subtree $\tilde{T} \in \mathcal{T}(x)$ is satisfied: If $\tilde{T}=T^{\prime}$ then the conditions are satisfied by construction and if $\tilde{T} \neq T^{\prime}$ then $\alpha_{\ell}\left(\tilde{T}, L^{\prime}\right) \leq \alpha_{\ell}\left(T-T^{\prime}, L^{\prime}\right)<\frac{k}{2}$ holds because $\tilde{T} \subseteq T-T^{\prime}$ and $\alpha_{\tilde{\ell}}\left(T-\tilde{T}, L^{\prime}\right)+\beta_{\tilde{\ell}}\left(T-\tilde{T}, L^{\prime}\right) \geq \alpha_{\tilde{\ell}}\left(T^{\prime}, L^{\prime}\right)+$ $\beta_{\tilde{\ell}}\left(T^{\prime}, L^{\prime}\right) \geq \frac{k}{2}$ because $T^{\prime} \subseteq T-\tilde{T}$.

Therefore, $x$ is $k$-centrum for $\tilde{\ell}$ and hence $\left(p^{*}, q^{*}\right)$ is an optimal solution of InvKC. 
Theorem 4.1 implies that an optimal solution of Relax $\left(T^{\prime}\right)$ is also optimal for InvKC. In the next step an optimal solution of $\operatorname{Relax}\left(P^{\prime}\right)$ is investigated in more detail. Obviously there exists an optimal solution $\left(p^{*}, q^{*}, L^{*}\right)$ such that if the length of edge $e=(a, b)$ is changed then the length of each edge on $P(x, a)$ is modified by the maximal allowable value because every modification can be shifted in direction of vertex $x$. This means that the lengths of edges near to $x$ are modified before those of edges that are farther away. A solution with this property is called nice. Moreover, the following lemma states that there exists an optimal solution such that there is a vertex $v \in V$ whose modified distance to $x$ is equal to $L^{*}$ and the modification on the path $P(x, v)$ has the following special form: There exists a vertex $v^{\prime}$ on path $P(x, v)$ and the lengths of all edges from $x$ to $v^{\prime}$ are maximally changed (i.e., modified until the bound is reached) while the lengths of all other edges on path $P(x, v)$ are not changed at all.

Lemma 4.2. Assume that $T^{\prime}$ violates the optimality criterion for $\ell$. Then there exists an optimal solution $\left(p^{*}, q^{*}, L^{*}\right)$ of Relax $\left(T^{\prime}\right)$ and vertices $v \in V$ and $v^{\prime} \in P(x, v)$ such that $d_{\tilde{\ell}}(x, v)=L^{*}=d_{\ell-u^{-}}\left(x, v^{\prime}\right)+d_{\ell}\left(v^{\prime}, v\right)$ if $v \in T^{\prime}$ and $d_{\tilde{\ell}}(x, v)=L^{*}=d_{\ell+u^{+}}\left(x, v^{\prime}\right)+d_{\ell}\left(v^{\prime}, v\right)$ if $v \in T-T^{\prime}$ where $\tilde{\ell}=\ell+p^{*}-q^{*}$.

Proof. Consider a nice optimal solution $\left(p^{*}, q^{*}, L^{*}\right)$ and assume that the property stated in the lemma is not true. Then for every vertex $v \in V$ with $d_{\tilde{\ell}}(x, v)=L^{*}$ there exists exactly one edge $e(v)$ that lies on the path $P(x, v)$ which is only partially modified, i.e., $0<p_{e(v)}^{*}<u_{e(v)}^{+}$or $0<q_{e(v)}^{*}<u_{e(v)}^{-}$. Let

$$
X_{1}=\left\{e(v) \in T^{\prime} \mid v \in T^{\prime} \text { with } d_{\tilde{\ell}}(x, v)=L^{*}\right\}
$$

and $X_{2}$ is defined for $T-T^{\prime}$ in an analogous way.

Assume that $\left|X_{1}\right|>\left|X_{2}\right|$. If the modification of every edge in $X_{1}$ is reduced by $\epsilon$ and the modification of every edge in $X_{2}$ is increased by $\epsilon$ then the resulting solution is still nice and feasible for $L^{*}+\epsilon$ and has less cost than the original solution which leads to a contradiction. For $\left|X_{1}\right|<\left|X_{2}\right|$ an analogous argument holds. Hence, it remains to consider the case $\left|X_{1}\right|=$ $\left|X_{2}\right|$. Then the same shift operation as mentioned above is used until at least one weight modification reaches 0 or its upper bound. This procedure yields a nice and optimal solution with the property mentioned in the lemma.

Lemma 4.2 immediately implies that we may restrict the set of potential optimal values for $L$ such that $L \in \mathcal{L}$ with

$$
\mathcal{L}=\left\{d_{\ell-u^{-}}\left(x, v_{1}\right)+d_{\ell}\left(v_{1}, v\right) \mid v, v_{1} \in T^{\prime}\right\} \cup\left\{d_{\ell+u^{+}}\left(x, v_{1}\right)+d_{\ell}\left(v_{1}, v\right) \mid v, v_{1} \in T-T^{\prime}\right\} .
$$


Hence, we have to solve $\min _{L \in \mathcal{L}} \xi(L)+\eta(L)$ where $\xi(L)$ denotes the minimum cost of an edge length modification such that there are at most $\frac{k}{2}$ vertices in $T^{\prime}$ having a distance to $x$ of more than $L$ and $\eta(L)$ denotes the minimum cost of an edge length modification such that there are at least $\frac{k}{2}$ vertices in $T-T^{\prime}$ having a distance to $x$ of at least $L$. Hence, for each $L \in \mathcal{L}$ we have to solve two independent subproblems, one in $T^{\prime}$ and the other one in $T-T^{\prime}$. Each subproblem can be solved in $\mathcal{O}\left(n k^{2}\right)$ time by using a bottom-up dynamic programming approach. Hence, we get

Theorem 4.3. The inverse unit-weight $k$-centrum problem can be solved in $\mathcal{O}\left(n^{3} k^{2}\right)$ time

Shortening the length of edges in $T^{\prime}$. A vertex whose distance to $x$ is more than $L$ is called remote. Assume that $L \in \mathcal{L}$ is fixed and we have to shorten the lengths in $T^{\prime}$ such that there are at most $\frac{k}{2}$ remote vertices.

The main idea is to use the special structure of nice solutions. For each vertex $v \in V$ compute the minimum cost $\kappa\left(T_{v}, r\right)$ such that there are at most $r$ remote vertices in $T_{v}$ (for $r=0, \ldots, \frac{k}{2}$ ). Then $\xi(L)$ is equal to $\kappa\left(T_{x}, \frac{k}{2}\right)$.

Let us start with a leaf $v \in V$. Then $\kappa\left(T_{v}, r\right)=0$ for all $r \geq 1$. If $r=0$ then we have to distinguish whether $v$ is a remote vertex before any modification or not. If $d_{\ell}(v, x) \leq L$ then $\kappa\left(T_{v}, 0\right)=0$. However, if $d_{\ell}(v, x)>L$ then $\kappa\left(T_{v}, 0\right)=d_{\ell}(v, x)-L$ if $d_{\ell-u^{-}}(v, x) \leq L$ and otherwise $\kappa\left(T_{v}, 0\right)=\infty$ because the bound constraints do not allow to remove the remoteness of $v$.

In the next step consider two children $v_{1}$ and $v_{2}$ of a vertex $v \in V$. Observe that there are at most $r$ remote vertices in $T_{v_{1}} \cup T_{v_{2}}$ if and only if there are at most $r_{1}$ remote vertices in $T_{v_{1}}$ and at most $r-r_{1}$ remote vertices in $T_{v_{2}}$ for some $0 \leq r_{1} \leq r$. Since we may restrict ourselves to nice solutions there exists a $0 \leq r_{1} \leq r$ such that solutions that are associated with $\kappa\left(T_{v_{1}}, r_{1}\right)$ and $\kappa\left(T_{v_{2}}, r-r_{1}\right)$ yield an optimal solution with at most $r$ remote vertices in $T_{v_{1}} \cup T_{v_{2}}$. Hence, we can compute $\kappa\left(T_{v_{1}} \cup T_{v_{2}}, r\right)$ in $\mathcal{O}(r)$ time.

Now assume that we already know $\kappa\left(\bigcup_{v_{i} \in \Gamma(v)} T_{v_{i}}, r\right)$ for $r=0, \ldots, \frac{k}{2}$ where $\Gamma(v)$ denotes the set of all children of $v$. Then $\kappa\left(T_{v}, r\right)=0$ for all $r \geq\left|T_{v}\right|$. If $r=\left|T_{v}\right|-1$ then vertex $v$ is the only one that is not allowed to be remote. Hence, this case is equivalent to the case for $v$ is a leaf and $r=0$. Finally, $\kappa\left(T_{v}, r\right)=\kappa\left(\bigcup_{v_{i} \in \Gamma(v)} T_{v_{i}}, r-1\right)$ for all $r \leq\left|T_{v}\right|-2$.

We have constructed a dynamic programming algorithm. Each $\kappa\left(T_{v}, r\right)$ can be computed in $\mathcal{O}(\operatorname{deg}(v) r)$ time. Hence, the total running time is $\mathcal{O}\left(n k^{2}\right)$. 
Increasing the lengths of edges in $T-T^{\prime}$. The task of increasing the lengths such that there are at least $\frac{k}{2}$ vertices whose distance to $x$ is at most $L \in \mathcal{L}$ can also be solved in $\mathcal{O}\left(n k^{2}\right)$ time by using an analogous dynamic programming approach.

\section{Conclusion}

This paper deals with changing edge length within certain bounds such that a prespecified vertex becomes ordered median. We prove NP-hardness even if the ordered median problem is convex and the graph is a tree. The problem remains NP-hard if all vertex weights are equal to 1 or if the $k$-centrum problem, a special ordered median problem, is considered. On the other hand, a polynomial time algorithm for the inverse unit-weight $k$-centrum problem on trees is developed.

It would be interesting to consider further special cases of convex inverse ordered median problems. Another direction of future research is the investigation of non-convex ordered median problems.

\section{References}

[1] Rainer E. Burkard, Carmen Pleschiutschnig, and Jianzhong Zhang. Inverse median problems. Discrete Optim., 1(1):23-39, 2004.

[2] Rainer E. Burkard, Carmen Pleschiutschnig, and Jianzhong Zhang. The inverse 1-median problem on a cycle. Discrete Optim., 5(2):242-253, 2008 .

[3] Rainer Ernst Burkard, Mohammadreza Galavii, and Elisabeth Gassner. The inverse fermat-weber problem. Technical Report 2008-14, Graz University of Technology, 2008. http://www.math.tugraz.at/fosp/ pdfs/tugraz_0107.pdf.

[4] M. C. Cai, X. G. Yang, and J. Z. Zhang. The complexity analysis of the inverse center location problem. J. Global Optim., 15(2):213-218, 1999 .

[5] Patricia Dominguez-Marin. The Discrete Ordered Median Problem: Models and Solution Methods, volume 15 of Combinatorial Optimization Series. Springer), Netherland, 2003. 
[6] Michael R. Garey and David S. Johnson. Computers and intractability. W. H. Freeman and Co., San Francisco, Calif., 1979. A guide to the theory of NP-completeness, A Series of Books in the Mathematical Sciences.

[7] Elisabeth Gassner. The inverse 1-maxian problem with edge length modification. J. Comb. Optim., 16(1):50-67, 2008.

[8] A. J. Goldman. Optimal center location in simple networks. Transportation Sci., 5:212-221, 1971.

[9] Clemens Heuberger. Inverse combinatorial optimization: a survey on problems, methods, and results. J. Comb. Optim., 8(3):329-361, 2004.

[10] Jörg Kalcsics, Stefan Nickel, Justo Puerto, and Arie Tamir. Algorithmic results for ordered median problems. Oper. Res. Lett., 30(3):149-158, 2002.

[11] Stefan Nickel and Justo Puerto. A unified approach to network location problems. Networks, 34(4):283-290, 1999. Centrality concepts in network location.

[12] Arie Tamir. The $k$-centrum multi-facility location problem. Discrete Appl. Math., 109(3):293-307, 2001. 